\title{
Guidance-Based Quantification of Arm Impairment Following Brain Injury: A Pilot Study
}

\author{
David J. Reinkensmeyer, Member, IEEE, Julius P. A. Dewald, and William Zev Rymer, Member, IEEE
}

\begin{abstract}
This paper reports the design and preliminary testing of a device for evaluating arm impairment after brain injury. The assisted rehabilitation and measurement (ARM) Guide is capable of mechanically guiding reaching and retrieval movements across the workspace and of measuring constraint forces and range of motion during guidance. We tested the device on four hemiplegic brain-injured individuals and four unimpaired control subjects. During guided movement, the brain-injured subjects generated distinct spatial patterns of constraint force with their impaired arms that were consistent with the standard flexion and extension "synergies" described in the clinical literature. In addition, the impaired arms exhibited well-defined workspace deficits as measured by the ARM Guide. These results suggest that constraint force and range of motion measurements during mechanically guided movement may prove useful for precise monitoring of arm impairment and of the effects of treatment techniques targeted at abnormal synergies and workspace deficits.
\end{abstract}

Index Terms-Biological motor systems, biomechanics, motion measurement, nervous system, robots.

\section{INTRODUCTION}

A PPROXIMATELY 400000 individuals survive a stroke in the United States each year [1]. Of these, over 50\% incur chronic motor disability [2]. Tens of thousands more people annually experience traumatic brain injuries that cause motor deficits [3]. Currently, little mechanical sensing and actuation technology is used in the clinical evaluation of motor impairments after such brain insult. Imaging technology is used to localize central nervous system (CNS) lesions, and electrophysiological technology, such as electromyogram (EMG) and H-reflex testing, is sometimes used to quantify muscle and reflex activation. However, evaluation of the biomechanical effects of neural damage relies to a large degree on visual and haptic observations by therapists and

Manuscript received October 1, 1997; revised May 7, 1998. This work was supported by NIH-F32HD08067, NIDRR-H133P20016, and the Ralph and Marian C. Falk Medical Research Trust.

D. J. Reinkensmeyer was with the Department of Physical Medicine and Rehabilitation, Northwestern University Medical School, and the Sensory Motor Performance Program, Rehabilitation Institute of Chicago, Chicago, IL 60611 USA. He is now with the Department of Mechanical and Aerospace Engineering, University of California at Irvine, Irvine, CA 92697 USA.

J. P. A. Dewald is with the Department of Physical Medicine and Rehabilitation, Northwestern University Medical School, the Programs in Physical Therapy, Northwestern University, and the Sensory Motor Performance Program, Rehabilitation Institute of Chicago, Chicago, IL 60611 USA.

W. Z. Rymer is with the Department of Physiology, Northwestern University, the Department of Physical Medicine and Rehabilitation, Northwestern University Medical School, and the Sensory Motor Performance Program, Rehabilitation Institute of Chicago, Chicago, IL 60611 USA.

Publisher Item Identifier S 1063-6528(99)01170-2. physicians. Appropriately designed machines could enhance subjective, haptic evaluation. In particular, machine-assisted evaluation might improve the objectivity, sensitivity, and diagnostic power of the hands-on, clinical exam [4]-[7].

By "appropriately designed" machines we mean machines targeted at the distinctive motor impairments that arise after brain injury. These impairments include weakness [8], [9], contracture [10], spasticity [11], and incoordination [12], [13]. Several mechanical devices have been developed for quantifying weakness, contracture, and spasticity, some of which are routinely used in clinics. For example, dynamo meters are available for measuring weakness [14] and goniometers for measuring contracture [15]. Devices for measuring spasticity at the elbow, ankle, and other joints are also under development [16], [17] but have not yet found widespread use.

A shortcoming of current quantification devices is that they typically focus on single-joint movement while motor deficits after brain injury often involve impaired multijoint coordination. In particular, arm and leg movement after brain injury often appears to be limited by relatively tight, stereotypical coupling of motion at adjacent joints, induced by abnormal coactivation of muscles acting at the adjacent joints [18]-[20]. This coupling is clinically referred to as an "abnormal synergy." Abnormal synergies were described in detail by Brunnstrom [21] and are typically grouped into flexion and extension synergies for the arm and leg (Table I). A key functional consequence of abnormal synergies is thought to be a decreased capacity to move the affected limb to desired locations in space [21].

Currently, few quantitative methods are available to characterize abnormal synergies and their effects on a limb's workspace. In rehabilitation practice abnormal synergies are typically evaluated qualitatively based on the descriptive studies by Brunnstrom. Quantitative scoring in the clinic is sometimes achieved using the Fugl-Meyer motor performance exam [22], which is a cumulative score that coarsely grades a patient's ability to move joints independently or in various patterns based on Brunnstrom's descriptions (see Table I). As for technology-based measurement, multiple-muscle EMG's combined with multiaxial force sensing have been used to characterize abnormal muscle synergies [23], but the complexity of these procedures would appear to hinder their routine clinical use. Isometric, multiaxis force target matching is another technique which in preliminary studies has shown promise for quantifying the multijoint effects of abnormal synergies on directional weakness [24]. However, important unanswered questions remain concerning how to quantify 
TABLE I

Summary of Subject Characteristics and Fugl-Meyer Motor Performance Exam Scores for the Impaired ARM's of the Four Brain-Injured Subjects. $0=$ the Specific Detail Cannot Be

Performed at all; $1=$ the Detail Can Be Performed only Partly; 2 $=$ The Detail Is Performed Faultlessly. The Individual Joint Components of THe Flexion and Extension Synergies, Derived from Brunnstroms Work, Are Shown in Part II of the Table

\begin{tabular}{|c|c|c|c|c|c|}
\hline & Subject: & A & B & C & D \\
\hline \multirow[t]{4}{*}{ Subject Characteristics: } & Age: & 24 & $\overline{52}$ & $\overline{74}$ & 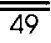 \\
\hline & Type of brain injury: & TBI & CVA & CVA & TBI \\
\hline & Since injury (years): & 2 & 2 & 25 & 16 \\
\hline & Side of paresis: & $r$ & $r$ & $r$ & 1 \\
\hline \multicolumn{6}{|c|}{ Fugl-Meyer Exam Upper Exfremity Scores: } \\
\hline I. Reflex activity & out of possible 4: & 4 & 4 & 4 & 4 \\
\hline II. Movement in synergy & out of possible 18: & 13 & 17 & 10 & 7 \\
\hline \multirow{9}{*}{$\begin{array}{c}\text { individual componen } \\
\text { flexion synergy: } \\
\text { extension synergy: }\end{array}$} & shoulder retraction & 2 & 2 & 2 & 1 \\
\hline & olevation & 2 & 2 & 2 & 1 \\
\hline & abduction & 1 & 2 & 2 & 1 \\
\hline & external rotation & 2 & 2 & 1 & 0 \\
\hline & & 2 & 2 & 2 & 2 \\
\hline & forearm supination & 0 & 2 & 0 & 1 \\
\hline & & 2 & 2 & 1 & 1 \\
\hline & elbow extension & 2 & 2 & 0 & 0 \\
\hline & forearm pronation & 0 & 1 & 0 & 0 \\
\hline III. Mixing synergies: & out of possible 6: & 3 & 2 & 0 & $\overline{0}$ \\
\hline IV. Out of synergy: & out of possible $6:$ & 3 & 0 & 0 & 0 \\
\hline V. Wrist and hand & out of possible 24: & 0 & 0 & 1 & 1 \\
\hline VI. Coordination/Speed & out of possible 6: & 1 & 0 & 1 & 0 \\
\hline Total: & & 24 & 23 & 16 & $\overline{\overline{12}}$ \\
\hline
\end{tabular}

abnormal synergies during dynamic movement and the effects of abnormal synergies on a limb's ability to move to different locations in space.

This paper describes a method and device designed to quantify both impaired multijoint coordination and workspace deficits during arm movement after brain injury. As a starting point for the device design, we hypothesized that abnormal synergies would appear as distinct patterns of constraint force at the hand during mechanically guided movement. In addition, we sought to test whether guided movement could be used to evaluate workspace deficits after brain injury. The device we have developed, called the "assisted rehabilitation and measurement (ARM) Guide," attaches to the patient's forearm and guides the arm along a linear path, measuring constraint forces and range of motion (Fig. 1). This paper reports pilot testing of the ARM Guide with four hemiplegic brain-injured subjects who were clinically identified as having abnormal synergetic patterns of arm movement, and four unimpaired control subjects. Portions of this work have been reported in abstract form [25] and [26].

\section{METHODS}

\section{A. Subject Selection}

The four control subjects were aged between 29-41 and had no known neurological deficits. Three of the control subjects were right-hand dominant, and one was left-hand dominant. As shown in Table I, the brain-injured subjects were aged between 24-74 and were at least two years post-brain injury. Three were right hemiplegic, and one was left hemiplegic. Table I
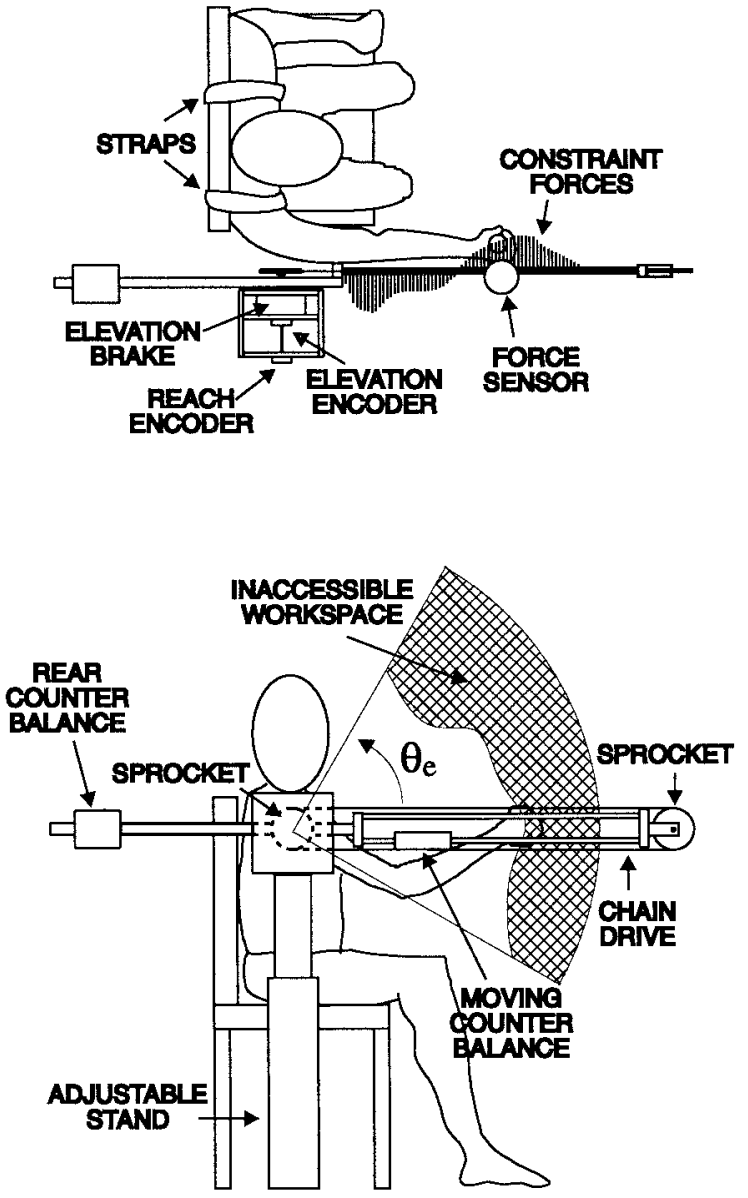

Fig. 1. The ARM Guide. The user moves the arm along a linear, counterbalanced constraint that can be oriented at different elevation angles $\theta_{e}$. Movement along the constraint and forces generated against the constraint are measured.

also shows the scores for the brain-injured subjects on the upper extremity portion of the Fugl-Meyer motor performance exam [22]. All subjects could perform active movement within synergy, but had limited active movement mixing synergies, or moving out of synergy (summarized in Fig. 2). Subjects $\mathrm{A}, \mathrm{C}$, and D could perform a small degree of gross flexion of the hand, but otherwise, like Subject B, had no residual hand or wrist function. None of the subjects had obvious evidence of abnormal synergetic movement or decreased active range-of-motion (ROM) of the arm on the side contralateral to the impaired arm, although it is possible that the motor capacity of the "unaffected" arm was also affected by the brain injury [27]. No subject exhibited cognitive deficits, neglect, or aphasia. The Institutional Review Board of Northwestern University, Evanston, IL, gave approval for all experiments. Subjects provided informed consent.

\section{B. Device Description}

The ARM Guide consists of an instrumented linear constraint that can be oriented in different directions across the subjects workspace (Fig. 1). The linear constraint is implemented using a three-splined steel shaft. The subject is attached to the ARM Guide using a custom splint that rides along the 


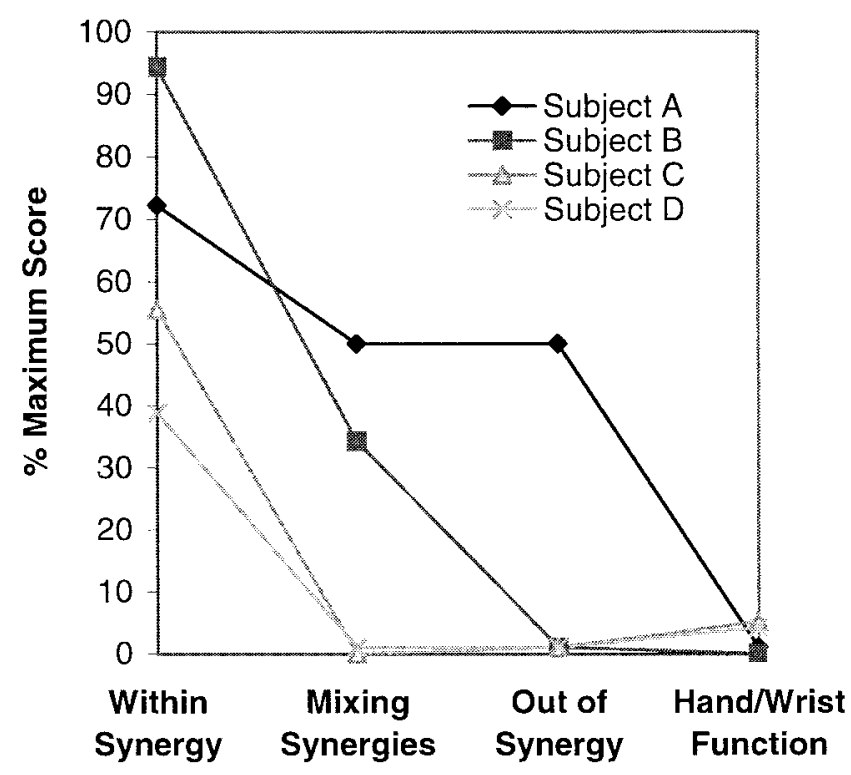

Fig. 2. Summary of movement ability of impaired arms of brain-injured subjects from Fugl-Meyer exam scores (see Table I).

linear constraint via a low friction, ball bearing, spline nut. The splint is allowed to rotate with respect to the constraint via a ball-bearing shaft holder. A six-axis force/torque sensor (ATI Industrial Automation) is interposed between the ball-bearing shaft holder and spline nut to measure the constraint forces generated by the subject against the ARM Guide (force/torque resolution $=0.5 \mathrm{~N} / 0.03 \mathrm{Nm}$ ).

The linear constraint can be oriented in different directions in the vertical plane referenced to the flexion/extension axis of rotation of the shoulder. In particular, the spline shaft is coupled to the (hollow) shaft of a computer-controlled magnetic particle brake (Placid Industries, Inc.). A backstop prevents the spline nut/handle from moving closer than $25 \mathrm{~cm}$ to the shaft of the brake. The linear constraint rotates about the brake axis to different elevation angles $\theta_{e}$. An optical encoder (U.S. Digital, Inc.) measures the elevation angle $\theta_{e}$ (resolution $=0.05^{\circ}$ ). The brake provides a scalable resistance torque between 3.0-150 lb-in, and can be used to hold the ARM Guide at a fixed elevation angle during arm movement. To lock the ARM Guide at a particular elevation angle, the experimenter manually orients the linear constraint using a visual display of the current elevation angle on the computer. When the sensed elevation angle is within $0.5^{\circ}$ of the desired elevation angle, the computer automatically sets the brake at its maximum resistance torque.

To measure position of the arm along the linear constraint, the spline nut is coupled to a zero-backlash cable chain looped around two aluminum sprockets, one at the distal end of the spline shaft, and one coincident with the shaft of the brake. This latter sprocket attaches to a steel shaft that feeds through the hollow shaft of the brake to a second encoder. This second encoder measures the position of the splint along the spline (i.e., via the chain drive) (resolution $0.1 \mathrm{~mm}$ ).

The ARM Guide is counterbalanced such that it remains at any position and orientation it is placed along the linear constraint, at any elevation angle. As a result, the subject experiences no static loading of the arm due to the weight of the ARM Guide. In particular, the handle assembly is counterbalanced using a moving counterbalance of equal weight (3.23 $\mathrm{kg}$ ) riding on a second, parallel spline shaft. The resulting constant, forward center of mass is counterbalanced by a rear counterbalance.

A key design criterion was that the ARM Guide must securely engage the subject's arm without requiring the subject to have the ability to grasp. Our solution was a custom splint consisting of a wooden cone handle and a lightly padded forearm brace. The brace is oriented so as to hold the forearm in $30^{\circ}$ of pronation, accommodating subjects with limited supination ability. The forearm is secured to the brace using two straps, thereby eliminating wrist movement. If the subject cannot voluntarily grasp the cone, the fingers are wrapped around the cone by the experimenter, and the cone is slid and locked so that it holds the dorsal surface of the hand and forearm flush against the forearm brace. The ARM Guide can be used to measure reaching readily with either left or right hand by reversing the orientations of both the linear constraint and the handle.

\section{Data Collection and Control}

The two encoders and the force/torque sensor are sampled at $250 \mathrm{~Hz}$ and read into an analog-to-digital (A/D) acquisition system of a Pentium-based personal computer. An 8-pt moving average is applied to the force-torque data. In addition, the computer subtracts the forces/torques produced by the weight of the user attachment interface from the total forces/torques to derive the forces the user generates. The computer can also give visual or auditory feedback about elevation angle, reach along the ARM Guide, constraint forces, and other measured variables.

\section{Experimental Protocol}

For the pilot test of the ARM Guide described here, the subjects sat in a chair next to the device. The subject's torso was securely strapped to the chair back across the clavicles and around the upper chest to minimize shoulder translation and torso movement. The arm was abducted $10-20^{\circ}$ to allow it to pass freely by the side of the body. The height of the ARM Guide and position of the chair were adjusted to align the approximate center of the head of the humerus, determined by palpation, with the axis of rotation of the ARM Guide.

For the experiments with the brain-injured subjects, the ARM Guide was locked at a series of elevations $\theta_{e}=\left\{0^{\circ}\right.$, $-30^{\circ},+30^{\circ},+60^{\circ}$ \} (see Fig. 3). The elevation angle $\theta_{e}=$ $0^{\circ}$ corresponded to horizontal. At negative elevation angles gravity assisted reaching, and at positive elevation angles gravity resisted reaching.

For reaching movements performed by the impaired arm, the subject started with the elbow flexed and reached out as far as possible, and as fast as possible, ten times. Instructions were given to "reach smoothly, as fast as possible" and to "try not to push against the ARM Guide." Peak movement velocity was reported verbally to the subject after each trial. Ten initial warm-up movements were performed at the $0^{\circ}$ elevation angle. 


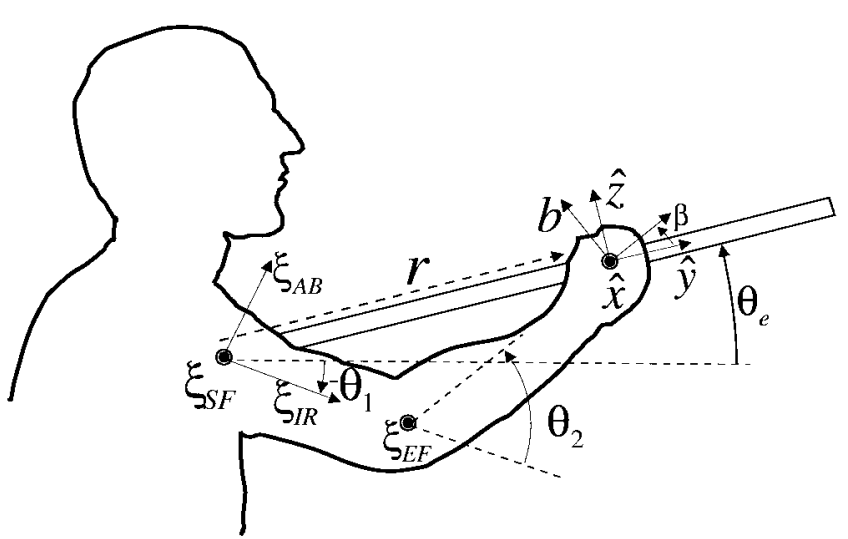

Fig. 3. Definition of coordinate systems, axes of rotation, and joint angles for the arm model used to infer shoulder joint torques. Definitions are shown for left arm. Medial direction is in positive direction.

A rest period of several minutes was given between movement sets at different elevation angles.

At the $-30^{\circ}$ elevation angle, retrieval-type movements were performed after the reaching movements. For these movements, the subject started with the elbow extended and moved back toward the backstop as fast as possible. Subjects were instructed to terminate movement before hitting the backstop if they had sufficient active ROM to reach the backstop.

The ARM Guide was further used to test for decreased passive ROM of the impaired arm at the smallest and largest elevation angles $\left(-30^{\circ}\right.$ and $\left.60^{\circ}\right)$. The subjects were asked to relax, and the experimenter moved the subject's arm using the moving counterbalance at a rate of approximately $20 \mathrm{~cm} / \mathrm{s}$ until a limit to movement was encountered. Five passive trials were performed.

The brain-injured subjects also performed movements with the contralateral arm. To approximate the dynamic conditions experienced by the impaired arm, subjects were instructed to attempt to match the average peak velocity achieved by the impaired arm at each elevation angle. The peak velocity achieved was verbally fed back after each trial, and subjects were instructed to adjust their movement speed to match the desired peak velocity.

To test the repeatability of the constraint force measurements, guided reaching movements by the impaired arms of brain-injured subjects $\mathrm{A}, \mathrm{B}$, and $\mathrm{C}$ were measured on three separate measurement sessions, spaced several weeks apart.

Guided movements were also performed by the four unimpaired, control subjects with both left and right arms. The purpose of these measurements was to check for left-right differences arising from hand-preference, to verify that the brain-injured subject's nonplegic arms generated ostensibly normal patterns of constraint force, and to determine the effects of movement speed and amplitude on constraint force patterns. Ten reaching movements at the $0^{\circ}$ elevation angle were performed at two different speeds, matched to the fastest and slowest speeds achieved by the impaired arms of the four brain-injured subjects $(20$ and $80 \mathrm{~cm} / \mathrm{s}$ ). Movements were also performed at both velocities at two different movement amplitudes (full ROM and $45 \mathrm{~cm}$-approximately half ROM).
Ten retrieval movements were also performed at the $-30^{\circ}$ elevation angle at $80 \mathrm{~cm} / \mathrm{s}$, at full and half ROM.

Measurement of movements by the control subjects at 80 $\mathrm{cm} / \mathrm{s}$, at the $0^{\circ}$ elevation angle, across the full ROM, were repeated three times on the subjects' nondominant arms to characterize measurement variability due to repositioning. For the second and third measurements the subjects were realigned with the ARM Guide.

\section{E. Data Analysis}

Movement trajectories along the ARM Guide were digitally differentiated to derive the reach velocity (Lanczos differentiating filter, 11 points, [28]). Force and position data were time-aligned at the time sample where the reach velocity exceeded a small arbitrary threshold greater than the resting noise of the velocity signal $(4 \mathrm{~cm} / \mathrm{s})$. The time-aligned force, position, and velocity trajectories were ensemble averaged for statistical comparison.

Endpoint forces and torques were related to joint torques at the elbow and shoulder using a simple kinematic model of the arm (Fig. 3). In particular, the constraint forces were related to the joint torques by the Jacobian of the arm model [29]

$$
\tau=J^{T} F_{b}
$$

where $\tau=\left[\begin{array}{llll}\tau_{S F} & \tau_{A B} & \tau_{I R} & \tau_{E F}\end{array}\right]^{T}$ is a vector of shoulder flexion, shoulder abduction, shoulder internal rotation, and elbow flexion torques defined about the axes $\xi_{S F}, \xi_{A B}, \xi_{I R}$, and $\xi_{E F}$ in Fig. 3 ; and $F_{b}=\left[\begin{array}{llllll}f_{x}^{b} & f_{y}^{b} & f_{z}^{b} & \tau_{x}^{b} & \tau_{y}^{b} & \tau_{z}^{b}\end{array}\right]^{T}$ is the vector of forces and torques in a body frame $b$ fixed to the subject's forearm at its connection to the ARM Guide. The shoulder internal rotation axis was defined relative to the shoulder abduction axis, which in turn was defined relative to the shoulder flexion axis.

Because the six-axis load cell rotates with the ARM Guide rather than the subject's forearm, its axes of measurement (defined as $\hat{x}, \hat{y}, \hat{z}$ in Fig. 3 ) were rotated by an angle $\beta$ with respect to the forearm body frame. In addition, because the subject's forearm was coupled to the ARM Guide through a rotary bearing, no torque could be generated about the $\hat{x}$ axis. The body wrench $F_{b}$ generated at the body frame affixed to the forearm is related to the load cell wrench $F$ by the adjoint matrix $A d_{\beta}$ that takes into account the rotation $\beta$ [29]

$$
F_{b}=A d_{\beta} F \text {. }
$$

Taken thus, the shoulder torques corresponding to constraint forces and torques measured at the force transducer are

$$
\begin{aligned}
\tau_{S F}= & \left(-l_{2} \sin \beta+l_{1} \sin \left(\theta_{2}-\beta\right)\right) f_{y} \\
& +\left(l_{2} \cos \beta+l_{1} \cos \left(\theta_{2}-\beta\right)\right) f_{z} \\
\tau_{A B}= & -\left(l_{1}+l_{2} \cos \theta_{2}\right) f_{x}+\sin \left(\theta_{2}-\beta\right) \tau_{y} \\
& +\cos \left(\theta_{2}-\beta\right) \tau_{z} \\
\tau_{I R}= & l_{2} \sin \theta_{2} f_{x}+\cos \left(\theta_{2}-\beta\right) \tau_{y}-\sin \left(\theta_{2}-\beta\right) \tau_{z}
\end{aligned}
$$

where $l_{1}$ and $l_{2}$ are the link lengths of the upper and lower arms, respectively. For each subject, the upper arm link length was measured as the distance between the visually estimated flexion/extension rotation centers of the shoulder and elbow, and the lower arm link length as the distance from the elbow 

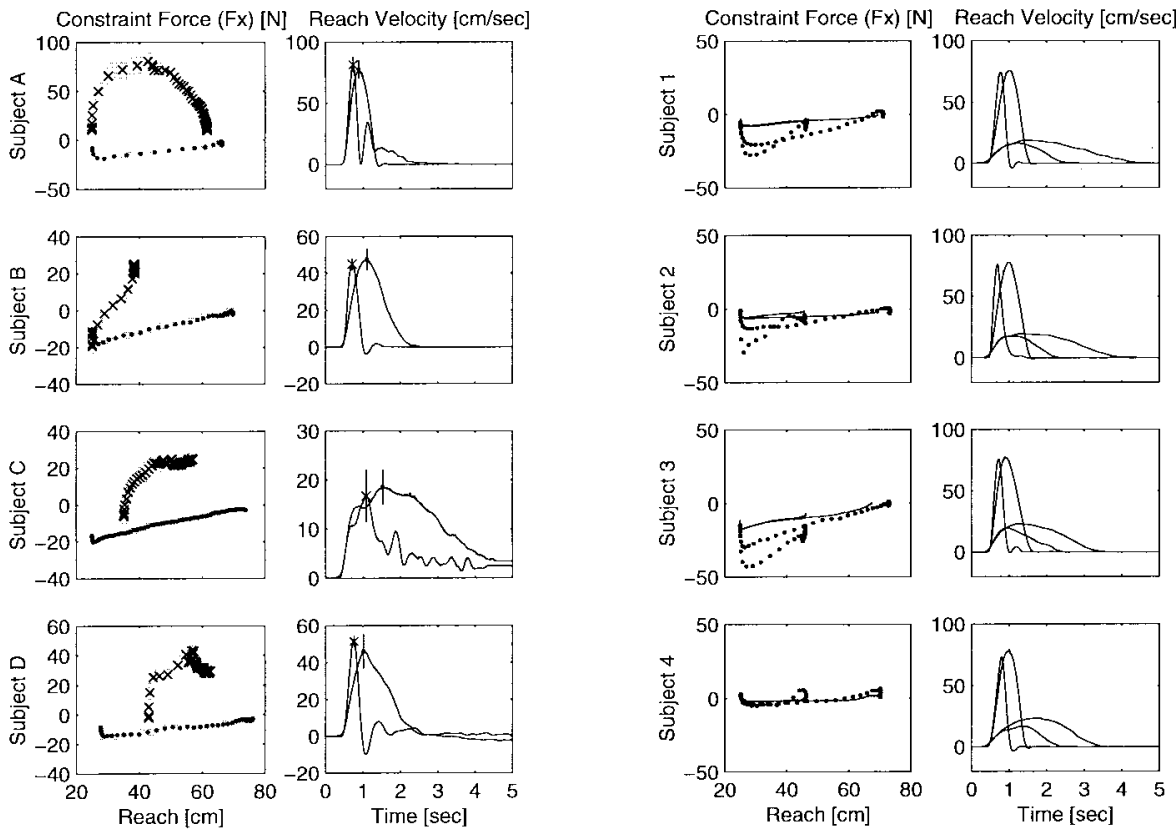

(a)

(b)

Fig. 4. (a) Average horizontal constraint force and reach velocity at $0^{\circ}$ elevation for ten reaches by the impaired $(x)$ and contralateral (.) arms of four hemiplegic brain-injured subjects. The $95 \%$ confidence intervals are shown for the horizontal constraint force and the peak velocity. Markers are $60 \mathrm{~ms}$ apart. (b) Average horizontal constraint force and reach velocity for ten reaches by the nondominant arm of four unimpaired, control subjects. Reaches were performed at a peak velocity of $20 \mathrm{~cm} / \mathrm{s}$ (solid lines) and $80 \mathrm{~cm} / \mathrm{s}$ (dotted lines) and at two movement amplitudes (full ROM and $45 \mathrm{~cm}$ ).

rotation center to the metacarpophalangeal joint. Note that (3) is valid only when the subject's arm is held statically along the ARM Guide, whereas (4) and (5) are valid during dynamic movement, since the subject's arm cannot move along the $\hat{x}$ axis or rotate around the $\hat{y}$ and $\hat{z}$ axes.

\section{RESULTS}

We examined impaired and contralateral arms of four hemiparetic subjects, and left and right arms of four unimpaired control subjects, performing a series of guided reaching and retrieval movements at different angles in the vertical plane. All of the hemiparetic subjects manifested standard abnormal synergies in the impaired arm, as judged by the Fugl-Meyer exam [22] (Table I, Fig. 2). We found that the impaired arms generated distinct patterns of constraint force during movement, particularly in the horizontal direction. Additionally, the impaired arms exhibited well-defined workspace deficits.

\section{A. Horizontal Constraint Force}

During reaching movements that began with the elbow flexed and proceeded with the hand extending out from the shoulder, the brain-injured subjects generated substantially different patterns of horizontal constraint force against the ARM Guide with the impaired and contralateral arms (Fig. 4). This force is defined as the force perpendicular to both the direction of movement and to the gravity vector (i.e., the force in the $\hat{x}$ direction in Fig. 3) and is defined positive in the medial direction, and negative in the lateral direction.

For the contralateral arms, the horizontal force was negative before reaching, became more negative at the initiation of reaching, and tended to increase slightly with increasing reach along the ARM Guide, reaching an average maximum across brain-injured and control subjects of $2.4 \mathrm{~N} \pm 4.6 \mathrm{SD}$ at the end of reach. In contrast, at the initiation of reaching by the impaired arms, horizontal constraint force became medially directed [Fig. 4(a)]. The mean peak medial constraint force achieved by the impaired arms was significantly greater than $10.0 \mathrm{~N}$ for all subjects (student's $t$-test, one sided, $p<0.05$ ). None of the contralateral arms of the hemiplegic subjects generated peak medial constraint forces significantly greater than $10.0 \mathrm{~N}(p>0.1)$. In addition, this $10.0 \mathrm{~N}$ cutoff was not exceeded by either the dominant or nondominant arm of the control subjects, at either the fast $(80 \mathrm{~cm} / \mathrm{s})$ or slow speeds $(20 \mathrm{~cm} / \mathrm{s})$, or for the full- or half-ROM reaching movements.

Despite the existence of a $10.0 \mathrm{~N}$ cutoff for the unimpaired arms, we did find significant left-right arm differences in horizontal constraint force for the control subjects, and a dependence of horizontal constraint force on movement speed [Fig. 4(b)]. For the same movement speed and amplitude, the horizontal constraint force followed the same pattern for the left and right arms, but tended to be offset in the medial direction for the dominant arm (mean offset across subjects and movement conditions $=3.2 \mathrm{~N} \pm 4.0 \mathrm{SD}$ ). For each subject, the horizontal constraint forces generated during the slow movements were approximately equal, regardless of the amplitude [Fig. 4(b)]. However, for the faster movements, the horizontal constraint forces were more laterally directed. The most laterally directed constraint forces were generated during the movements with the highest accelerations (i.e., the shorter movements performed at $80 \mathrm{~cm} / \mathrm{s}$ ), an effect opposite that seen with the impaired arms of the brain-injured subjects [Fig. 4(a)]. 
To test the repeatability of the horizontal constraint force measurement, we measured guided reaching movements by brain-injured subjects $\mathrm{A}, \mathrm{B}$, and $\mathrm{C}$ on three separate measurement sessions. The mean peak horizontal constraint force at the $0^{\circ}$ elevation angle, averaged across the three measurement sessions was $75.8 \pm 14.4 \mathrm{SD}(n=3)$ for subject $\mathrm{A}, 17.3 \pm 8.0$ for subject $\mathrm{B}$, and $22.5 \pm 5.8 \mathrm{~N}$ for subject $\mathrm{C}$. The mean peak horizontal constraint forces for the four control subjects across three measurement sessions were $5.0 \pm 1.9,1.9 \pm 0.6$, $2.7 \pm 0.8$, and $8.4 \pm 1.2 \mathrm{~N}$.

For the brain-injured subjects, the pattern of horizontal constraint force varied with the direction of movement across the workspace (Fig. 5). In particular, during movement initiation, the horizontal constraint force tended to rise more steeply in the medial direction with increasing elevation angle. In addition, the peak medial constraint force was significantly greater at the $+60^{\circ}$ versus $-30^{\circ}$ elevation angle for subjects $\mathrm{A}, \mathrm{B}$, and $\mathrm{C}(p<0.001$, one-sided student's $t$-test $)$, and at the $+30^{\circ}$ versus $-30^{\circ}$ for all subjects $(p<0.005)$. The horizontal constraint force produced by the contralateral arms did not vary substantially with elevation angle in comparison with the impaired arms (Fig. 5).

During retrieval type movements measured with the ARM Guide oriented at the $-30^{\circ}$ elevation angle, the impaired arm generated a different pattern of horizontal constraint force. For these movements, the subjects began with the arm extended and moved the hand toward the shoulder. All three subjects generated a significantly more laterally directed force than during reaching movements (Fig. 6). In contrast, for the contralateral arm, the horizontal constraint force pattern varied only slightly between reaching and retrieval movements.

\section{B. Other Components of Constraint Force; Joint Torques}

Three other constraint force/torque components were measured. The "vertical force" is defined as the force perpendicular to the ARM Guide in the sagittal plane (i.e., in the $\hat{z}$ direction in Fig. 3; note this "vertical" force is only truly vertical with respect to gravity when the ARM Guide is at $0^{\circ}$ elevation). This force tended to be negative and fairly constant throughout movement. No significant differences were found in the mean vertical constraint force between the impaired and unimpaired arms (mean for unimpaired arms at $0^{\circ}$ elevation $=-11.7$ $\mathrm{N} \pm 5.0 \mathrm{SD}$; mean for impaired arms at $0^{\circ}$ elevation $=$ $-9.1 \pm 12.6 \mathrm{SD})$.

Constraint torques were also measured against the ARM Guide around the $\hat{y}$ and $\hat{z}$ axes of the endpoint frame (Fig. 3). To interpret these endpoint torques, a simple biomechanical model of the arm was used to transform them, along with the mediolateral component of constraint force, into shoulder abduction/adduction and internal/external rotation torques about a frame at the shoulder [see Fig. 3 and (4) and (5)]. For the impaired arms, shoulder torques tended toward greater adduction and internal rotation during reaching, and greater abduction and external rotation during retrieval (Fig. 7). Peak shoulder adduction and internal rotation torques were significantly increased during reaching as compared with the contralateral side for the brain-injured subjects (Fig. 8, $p<0.001)$. Peak shoulder abduction and external rotation
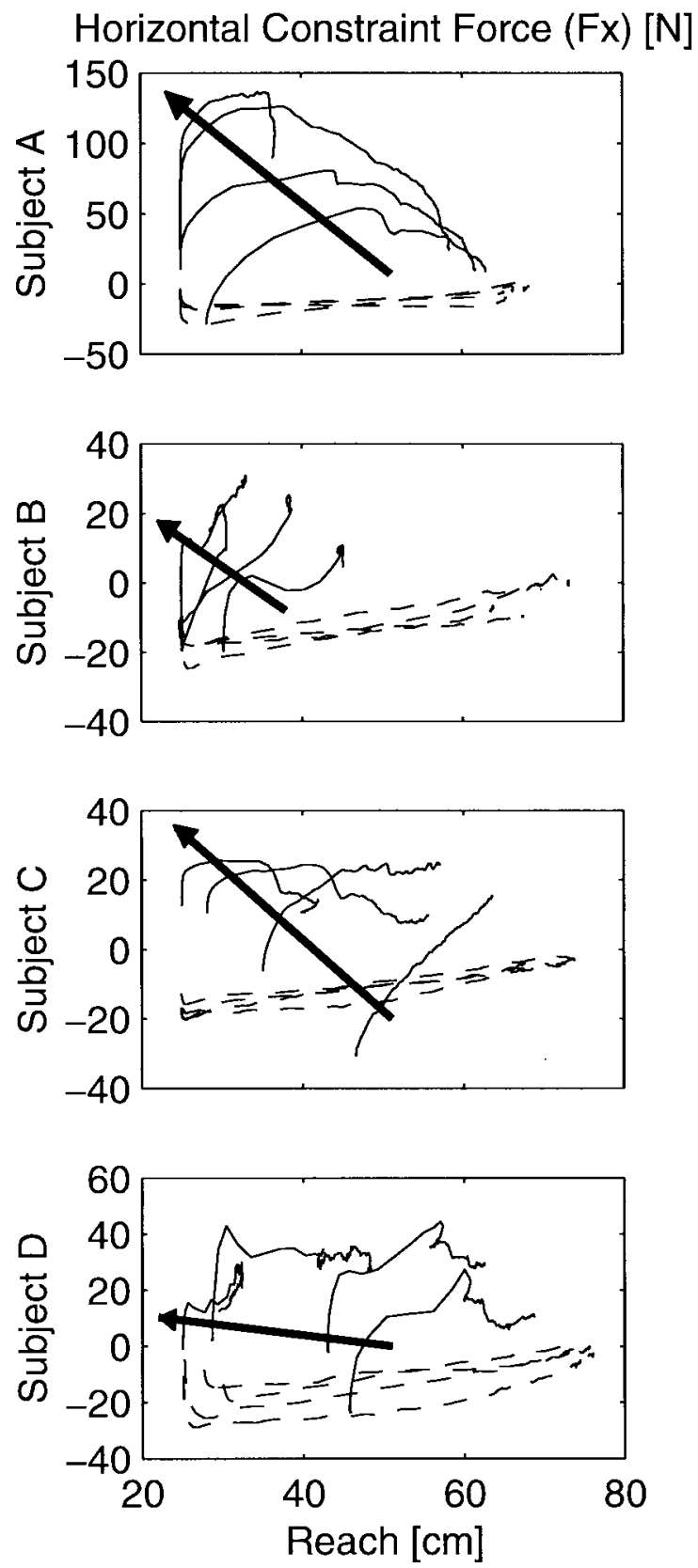

Fig. 5. Average horizontal constraint force for the impaired arm (solid) and contralateral arm (dashed) for elevations $\theta_{e}=\left\{-30-^{\circ}, 0^{\circ},+30^{\circ},+60^{\circ}\right\}$. Arrows identify increasing elevation angles.

torques were significantly increased during retrieval for all subjects $(p<0.05)$, although the increase in external rotation torque was small for subjects $\mathrm{C}$ and $\mathrm{D}$.

\section{Workspace Deficits}

Besides generating distinct patterns of constraint force against the ARM Guide, the brain-injured subjects had decreased active ROM along the ARM Guide with the impaired arm (Fig. 9). The impaired arms had significantly decreased active ROM with respect to the contralateral arms along at least two elevation angles $(p<0.001)$. As a result of these decreases, the percentage of inaccessible workspace area, measured as the difference between the impaired and 

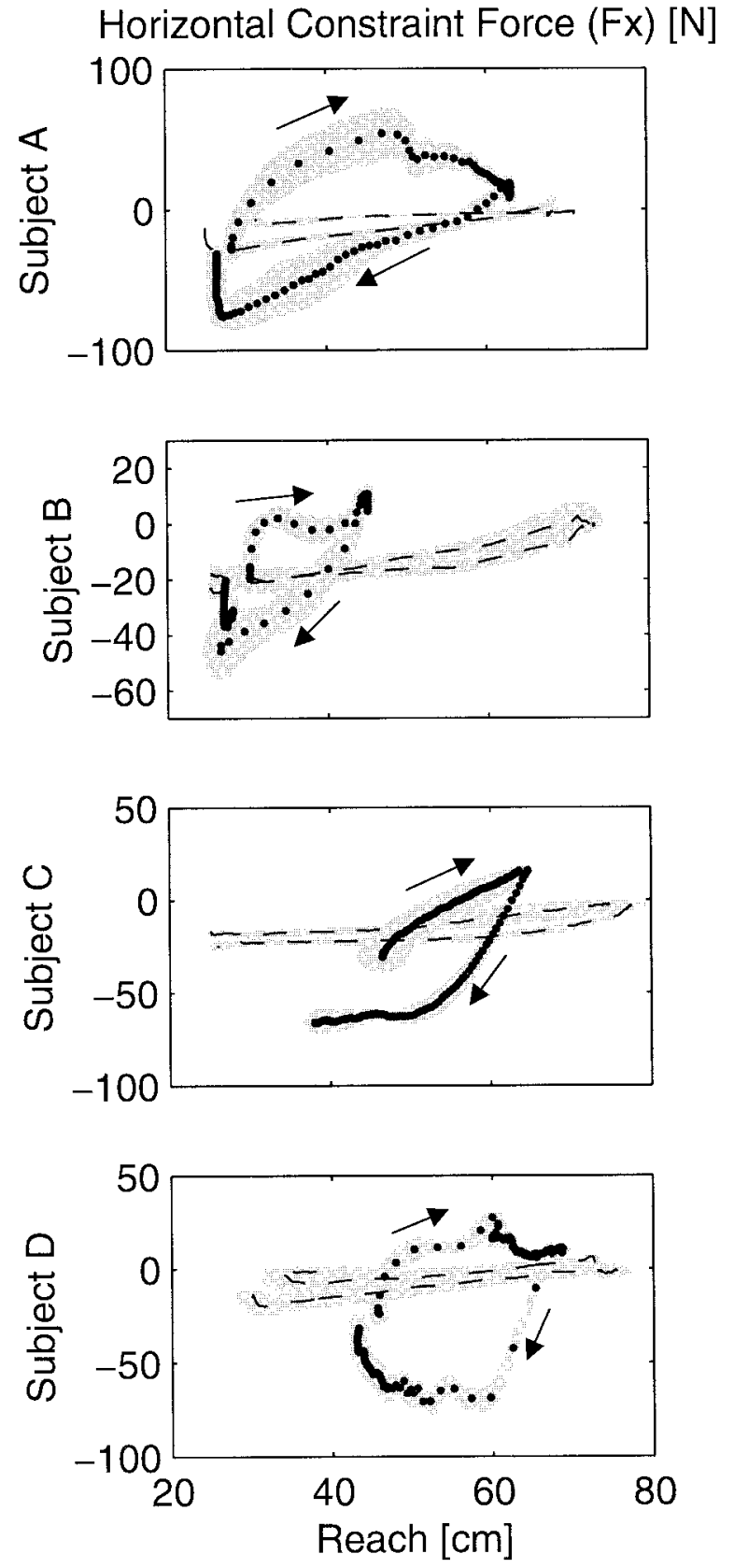

Fig. 6. Average horizontal constraint force for reaching and retrieval movements by the impaired (dotted) and contralateral arms (dashed) at $-30^{\circ}$ elevation angle. The direction of movement and 95\% confidence intervals are shown.

contralateral arms in the vertical plane, was approximately $24.8 \%$ for subject A, $71.2 \%$ for subject $\mathrm{B}, 47.6 \%$ for subject C, and $46.4 \%$ for subject D. For all subjects, the largest workspace deficits were seen at the $+60^{\circ}$ elevation angle.

The subjects' impaired arms exhibited slightly greater trial-to-trial variability in reaching than did the contralateral arms. The standard deviations of the maximum reach for the contralateral arms of the brain injured subjects were less than $1.5 \mathrm{~cm}$ for all elevation angles. The standard deviations for the impaired arms varied up to $3.0 \mathrm{~cm}$. at steeper elevation angles. The impaired arm standard deviations were significantly greater $(p<0.05)$ than the standard deviations of the contralateral arm for subject $\mathrm{A}$ at 30 and $60^{\circ}$ elevation angles, at no elevation angles for subject $\mathrm{B}$, at $-30^{\circ}, 30^{\circ}$, and $60^{\circ}$ for subject $\mathrm{C}$, and at no angles for subject $\mathrm{D}$. Thus, although some increased variability was seen in workspace measurements for the impaired arm, this variability was limited.

The ARM Guide was used to test for decreased passive ROM at the smallest and largest elevation angles by moving the subject's arm passively using the moving counterbalance. Subjects A and B had full passive ROM compared with the contralateral arm (Fig. 9). In contrast, subject C exhibited reduced ROM at both the smallest and largest elevation angles, generating a resisting force approximately five times greater than the contralateral arm at a similar position. Subject D also had reduced passive ROM at the steepest elevation angle, and experienced pain with passive shoulder flexion.

\section{Discussion AND CONCLUSIONS}

Our starting hypothesis for development of the ARM Guide was that abnormal synergies would manifest themselves as distinct patterns of constraint force during guided movement. Although we have tested a limited number of subjects, the pilot data support this hypothesis. In addition, the preliminary results support the feasibility of using mechanically guided movement as a means to quantify workspace deficits after brain injury.

\section{A. Constraint Force Measures of Impaired Coordination}

Distinct patterns of constraint force generation were seen in the horizontal direction for the brain-injured subjects. In particular, all of the impaired arms generated a more medially directed force during reaching, and a more laterally directed force during retrieval. These horizontal constraint force patterns are consistent with previous clinical [21] and experimental [23], [24] descriptions of abnormal synergies, and with the results of the Fugl-Meyer evaluations. A medial horizontal constraint force would be expected if the subjects were tending to internally rotate and/or adduct the shoulder during reaching. This motion is consistent with Brunnstrom's description of the extension synergy (Table I). A lateral constraint force would be expected if the subject were tending to externally rotate and/or abduct the shoulder during retrieval; i.e., the Brunnstrom flexion synergy. According to the Fugl-Meyer exam (Table I, Fig. 2), all of the brain-injured subjects were capable of moving within the extension and flexion synergies, but had limited ability to move out of synergy. These results support the hypothesis that the subjects used the extension and flexion synergies to propel the arm along the ARM Guide during reaching and retrieval.

The consistencies with previous descriptions of abnormal synergies were confirmed in shoulder torque space by mapping endpoint constraint forces/torques to joint torques with a model of the arm. In particular, shoulder abduction/adduction and internal/external shoulder torques, which do not contribute to movement along the ARM Guide, were increased in magnitude 

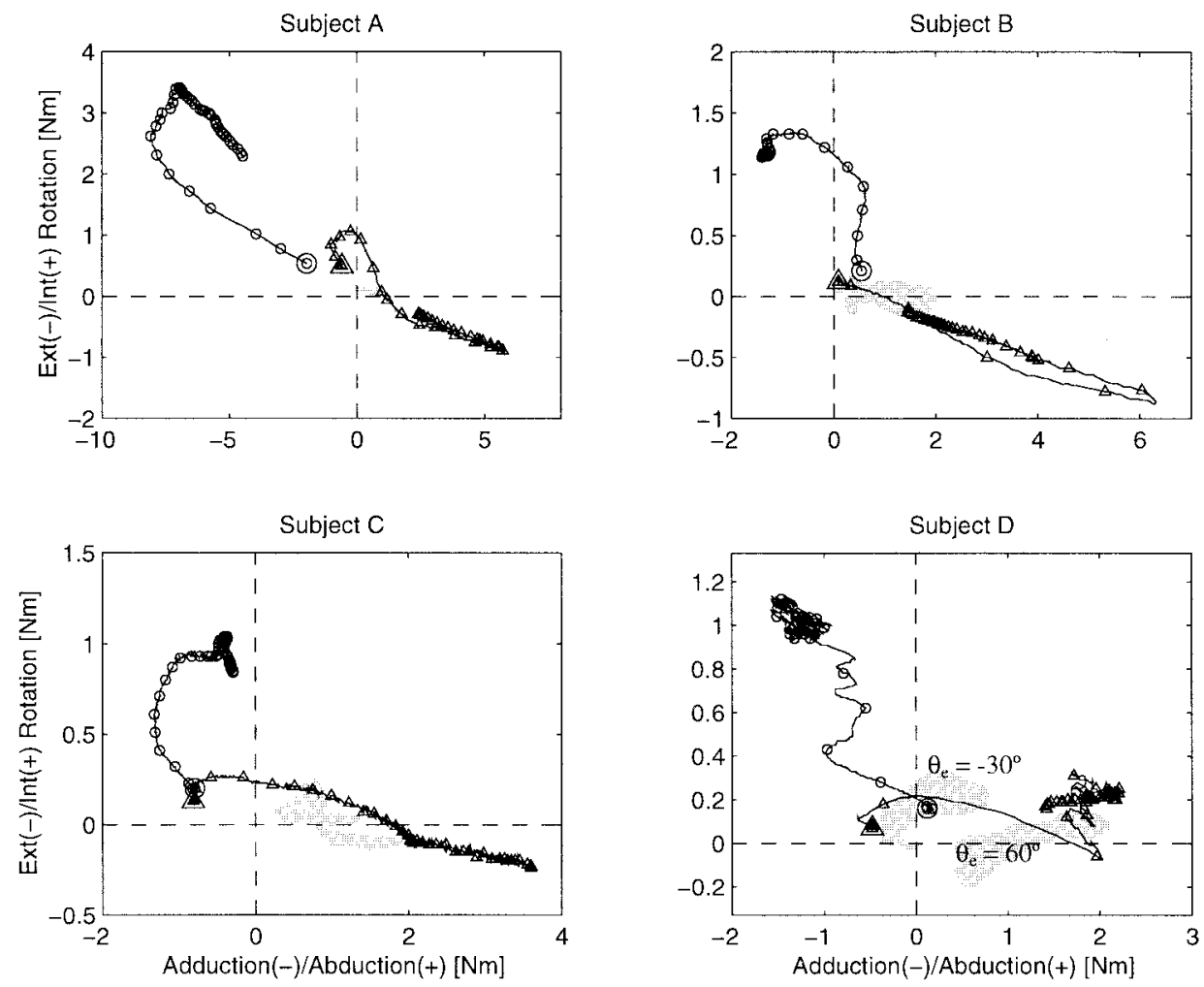

Fig. 7. Shoulder external/internal rotation and adduction/abduction torques for reaching movements at $+60^{\circ}(\mathrm{o})$ and retrieval movements at $-30^{\circ}(\Delta)$ elevation angles by the impaired arm. Markers are $100 \mathrm{~ms}$ apart; large markers show start of movement. Gray regions show $95 \%$ confidence intervals of shoulder torques for subject's contralateral arm at the same elevation angles. For subject D, the elevation angles corresponding to the confidence intervals are noted.

for the impaired arms. Shoulder adduction and internal rotation torques were increased during reaching, and shoulder abduction and external rotation were increased during retrieval movements. The smallest increases in shoulder external rotation torque during retrieval was seen for subjects C and D, consistent with the observation that these subjects also showed little or no ability to externally rotate the shoulder during the Fugl-Meyer exam (Table I).

Significant differences were not seen in the vertical constraint force. In general, both unimpaired and impaired arms apparently tended to rest on the ARM Guide during reaching, creating a negative vertical force. Based on currently available descriptions of the abnormal flexion and extension synergies, the synergies would not be expected to create distinct patterns of vertical constraint force: the vertical constraint force is directly related to the shoulder flexion/extension torque, and this degree of freedom has not been clinically or experimentally observed to be a strong component of either the flexion or extension synergy ([21], [24]).

In summary, based primarily on measurements in the horizontal direction, we believe that constraint force measurement during guided movement holds promise for quantitative evaluation of abnormal movement synergies. The initial direction and peak magnitude of horizontal constraint force in particular appear sensitive to the presence of abnormal synergies. By allowing quantification of what is typically described qualitatively, such measures may prove useful for more precise monitoring of the emergence and progression of abnormal coordination after brain injury, and of the effects of treatment techniques targeted at abnormal synergies.

\section{B. Limitations and Extensions of Constraint Force Measurements}

A requirement for use of the constraint force technique is the availability of an unimpaired limb for comparison, since unimpaired limbs also generate constraint forces during guided movement. In our study, even though instructions were given to the control subjects to minimize constraint force against the ARM Guide, they still generated a laterally directed horizontal force. We hypothesize that this laterally directed horizontal force is due to a mismatch between the geometry of the constraint imposed by the ARM Guide, and the geometry of normal reaching. For instance, the dorsum of the forearm does not typically move parallel to the path of the hand during normal reaching, although the ARM Guide enforces such a relationship. As a result of such small kinematic mismatches, accurate interpretation of abnormal patterns of constraint force depends on adequate characterization of patterns generated by unimpaired arms of similar physical dimensions. In addition, we hypothesize that accurate positioning of the subject is important for measurement reproducibility, since the positioning of the subject with respect to the device could potentially alter the pattern of kinematic mismatches. Finally, the constraint force patterns may depend on the subject's hand-dominance, and on the velocity trajectory of the movement, although the pilot data 

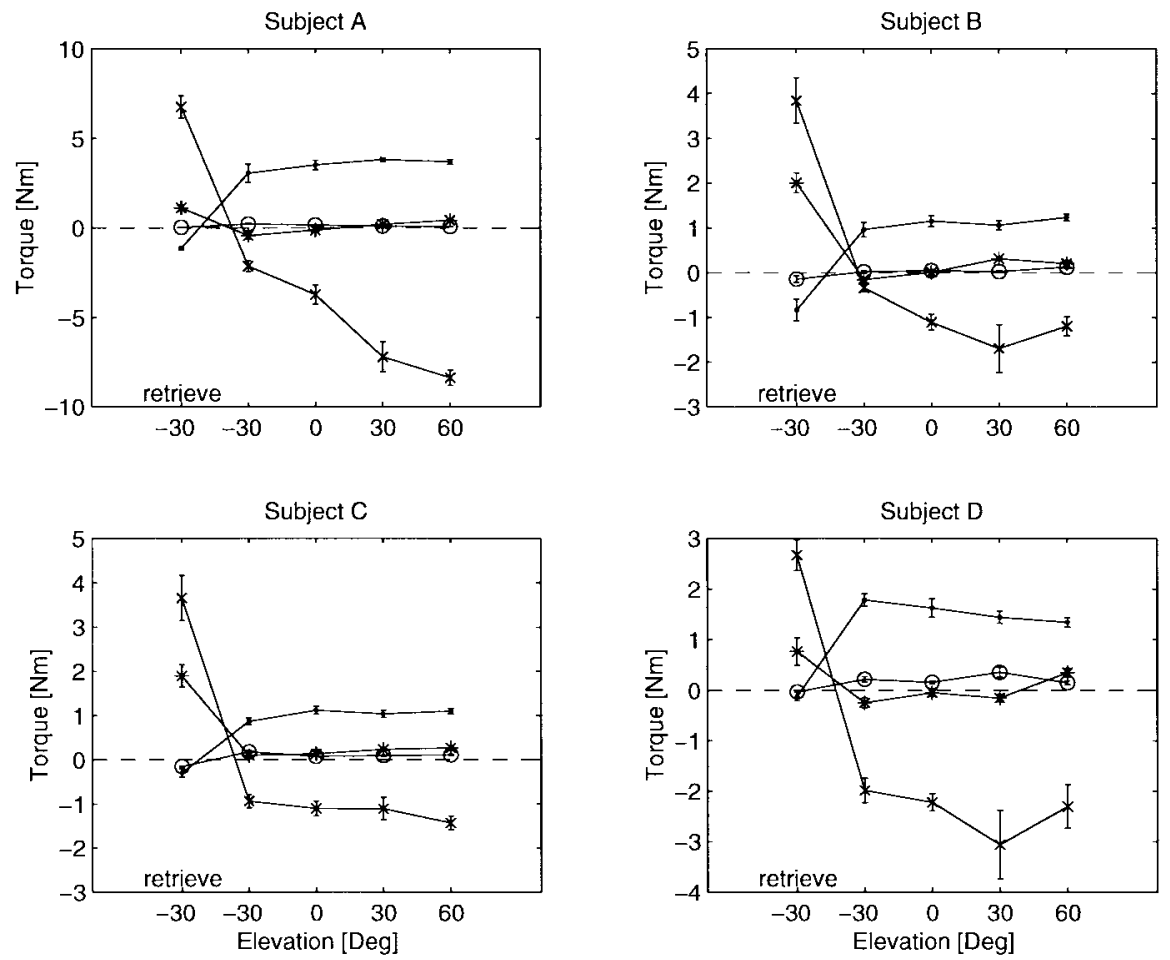

Fig. 8. Average peak shoulder torques and confidence intervals for reaching and retrieval movements by the impaired $[x, \bullet]$ and contralateral arms $\left[{ }^{*}\right.$, o] of the four brain-injured subjects. Peak adduction and internal rotation torques are shown for reaching movements at $\theta_{e}=\left\{-30^{\circ}, 0^{\circ},+30^{\circ},+60^{\circ}\right\}$, and peak abduction and external rotation torques are shown for retrieval movements at $\theta_{e}=-30^{\circ}$. Internal rotation and abduction are defined positive; external rotation and adduction are defined negative. $x$ : average peak abduction (or adduction for retrieval movement) torque for impaired arm; $*$ average peak abduction (or adduction) torque for contralateral arm; $\bullet$ : average peak internal (or external) rotation torque for impaired arm; o: average peak internal (or external) rotation torque for contralateral arm.

Subject A

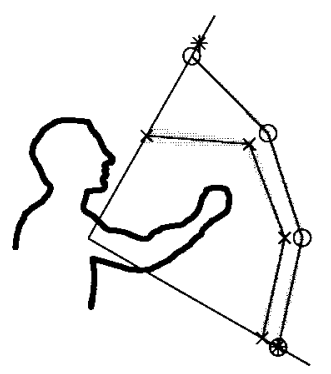

Subject B

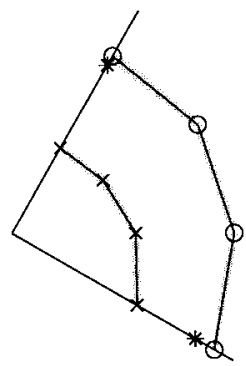

Subject C

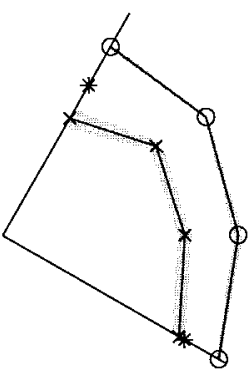

Subject D

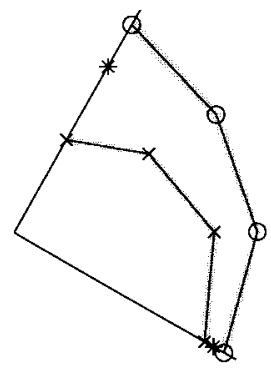

Fig. 9. Workspace boundaries $\pm 1 \mathrm{SD}$ for the four brain-injured subjects. $x$ : impaired arm; o: contralateral arm; $*$ passive ROM limit. The mean of the confidence intervals across subjects for the passive ROM measurements was $2.5 \mathrm{~cm}$. The radial bars are $80 \mathrm{~cm}$ long.

suggests it may be possible to bound these dependencies with respect to the differences in constraint force caused by the neurologic impairment.

It may be possible to extend the constraint force technique to individuals with other types of brain injuries, such as cerebral palsy, and also to other movements besides reaching and retrieval, such as leg movements or hand movements. In theory, the technique could be applied to any combination of body segments with at least two degrees of freedom coupled in an abnormal synergy by mechanically guiding movement along one of the degrees of freedom, and measuring the constraint forces generated by the other degree(s) of freedom.

\section{Guided Workspace Measures: Potential Use in Evaluating Movement Ability}

For the brain-injured subjects tested, the ARM Guide detected significant workspace deficits. Surprisingly, in light of the apparent large movement-to-movement variability observed subjectively in the clinic, the variability of maximum reach of the impaired arms was only slightly greater than the variability for the contralateral arms. Such low variability in guided workspace boundary measurement may allow sensitive monitoring of functional movement ability, provided the patient's arm can be reliably realigned with the ARM Guide during subsequent measurement sessions. A key question for fu- 
ture research is how well workspace measures correspond with the ability to perform various functional activities with the arm.

The ARM Guide could also potentially assist in diagnosing the causes of workspace deficits in individuals after brain injury. One potential cause is an involuntary limit to movement as a result of changed passive tissue properties (contracture), spasticity in antagonist muscles, or changes in the biomechanics of the shoulder (i.e., impaired gleno-humeral joint rhythm [30]). The subjects in this study experienced a well-defined limit in active ROM with the impaired arm, as judged by the variability of the endpoint of repeated reaching. The ARM Guide was used to determine if this measured limit of active movement corresponded to a limit in passive ROM. For the subjects in this study, decreased passive ROM matched decreased active ROM for only one subject at one elevation angle. Otherwise, the subjects had sufficient passive ROM to access difficult workspace areas, but could not enter those areas during voluntary movement, presumably because of an inability to generate needed forces. Future research will investigate in more detail the contributions of weakness and involuntary restraint mechanisms to workspace limitations by combining EMG measurements of arm muscles in conjunction with active and passive guided movements.

\section{Limitations and Extensions of Guided Workspace Measures}

The guided workspace measured with the ARM Guide may differ from the workspace achievable during unguided movement. That is, because the subject can generate components of force perpendicular to the ARM Guide and still move in the desired direction, he or she may be able to move farther with the ARM Guide than without it. However, the guided workspace still reflects the underlying movement ability of the subject, and thus may provide a simple means to evaluate movement performance. In addition, use of mechanical guidance has the potential advantage of allowing precise characterization of the passive mechanical properties of the limb along movement trajectories identical to those achieved during voluntary movement. Passive and active workspace deficits could potentially be measured in other planes of movement besides the vertical plane measured in this preliminary study.

An important, unanswered question concerning postbrain injury movement impairment is the relationship between weakness, abnormal synergies, and workspace deficits. If the abnormal extension synergy manifests itself as an increased medial constraint force, and a heightened extension synergy limits the workspace, one would predict higher medial constraint forces for greater workspace deficits. For the subjects we examined both the medial tendency in horizontal constraint force and workspace deficits increased at the highest elevation angle. Future research will also investigate in more detail the relationship between weakness and abnormal synergies, and the relative contribution of both of these impairments to limits in ROM across the workspace, and to the ability to perform functional tasks.

\section{E. Other Applications of Guided Movement: \\ Therapy for the Arm}

In conclusion, devices such as the ARM Guide might be useful not only as evaluation tools, but also as treatment tools. Real-time measurement of constraint force generation could be used to provide feedback to brain-injured individuals for training arm coordination. In addition, the ARM Guide is well suited to test the therapeutic effects of repeated, guided movement into different workspace regions. Guidance of active arm movement into inaccessible workspace areas is a technique used in manual therapy [31], and may aid neural recovery by allowing simultaneous sensory input and motor output not usually experienced following brain injury. Several research laboratories are currently developing robotic devices for providing therapeutic assistance to arm movement after brain injury [7]. A recent clinical trial of one of these devices suggests that robot-assisted movement can enhance motor recovery in stroke patients [32], [33]. It is the automation of manual therapy techniques, accompanied by the rational design of machine-based measurement techniques for evaluating motor impairments, that could provide key tools for advancing neurological rehabilitation research and practice in the future [34].

\section{ACKNOWLEDGMENT}

The authors wish to thank D. M. Lorber for assistance with software development for the ARM Guide.

\section{REFERENCES}

[1] G. E. Gresham, P. W. Duncan, and W. B. Stason, "Post-stroke rehabilitation," U.S. Dept. Health and Human Services, Agency for Health Care Policy and Research, Rockville, MD, AHCPR Publication No. 95-0662, 1995.

[2] G. E. Gresham, T. F. Phillips, P. A. Wolf, P. M. McNamara, W. B. Kannel, and T. R. Dawber, "Epidemiologic profile of long term disability in stroke: The Framingham study," Arch. Phys. Med. Rehab., vol. 60, pp. 487-491, 1979.

[3] B. H. Dobkin, Neurologic Rehabilitation. Philadelphia, PA: F. A. Davis, 1996.

[4] W. W. Tourtellotte and K. Syndulko, "Quantifying the neurologic examination: Principles, constraints, and opportunities," in Quantification of Neurologic Deficit, T. L. Munsat, Ed. Boston, MA: Butterworths, 1989, pp. 7-16.

[5] R. W. Bohannon, "Simple clinical measures," Phys. Ther., vol. 67, pp. 1846-1850, 1987.

[6] American Physical Therapy Association, Neurology Section, "Special topic issue on objective assessment of the neurologic patient," Neurol. Rep., vol. 16, 1992.

[7] D. J. Reinkensmeyer, N. Hogan, H. I. Krebs, S. L. Lehman, and P. S Lum, "Rehabilitators, robots, and guides: New tools for neurological rehabilitation," in Biomechanics and Neural Control of Movement, J. Winters and P. Crago, Eds. New York: Springer-Verlag, 1998.

[8] S. C. Gandevia, "Strength changes in hemiparesis: Measurements and mechanisms," in Spasticity: Mechanisms and Management, A. F. Thilmann, D. J. Burke, and W. Z. Rymer, Eds. Berlin, Germany: Springer-Verlag, 1993, pp. 111-122.

[9] D. Bourbonnais and S. Vanden Noven, "Weakness in patients with hemiparesis," Amer. J. Occupational Ther., vol. 43, pp. 313-319, 1989.

[10] N. J. O'Dwyer, L. Ada, and P. D. Neilson, "Spasticity and muscle contracture following stroke," Brain, vol. 119, pp. 1737-1749, 1996.

[11] A. F. Thilmann, D. J. Burke, and W. Z. Rymer (Eds.), Spasticity: Mechanisms and Management. Berlin, Germany: Springer-Verlag, 1993.

[12] D. Bourbonnais, S. Vanden Noven, and R. Pelletier, "In coordination in patients with hemiparesis," Canadian J. Public Health. Revue Canadienne de Sante Publique, vol. 83, suppl. 2, pp. S58-S63, 1992.

[13] M. F. Levin, "Interjoint coordination during pointing movements is disrupted in spastic hemiparesis," Brain, vol. 119, pp. 281-293, 1996.

[14] R. W. Bohannon, "Is the measurement of muscle strength appropriate in patients with brain lesions? A special communication," Phys. Ther., vol. 69, pp. 225-236, 1989.

[15] S. B. O'Sullivan and T. J. Schmitz, Physical Rehabilitation: Assessment and Treatment, 2nd ed. Philadelphia, PA: F. A. Davis, 1988. 
[16] R. T. Katz and W. Z. Rymer, "Spastic hypertonia: Mechanisms and measurement," Arch. Phys. Med. Rehab., vol. 70, pp. 144-155, 1989.

[17] J. R. Engsberg, K. S. Olree, S. A. Ross, and T. S. Park, "Quantitative clinical measure of spasticity in children with cerebral palsy," Arch. Phys. Med. Rehab., vol. 77, pp. 594-599, 1996.

[18] O. Foester, "Motorische felder und bahnen," in Handbuch der Neurologie, Bumke and O. Foester, Eds. Berlin, Germany: Springer-Verlag, 1936, vol. 6, pp. 1-357.

[19] T. E. Twitchell, "The restoration of motor function following hemiplegia in man," Brain, vol. 74, pp. 443-480, 1951.

[20] E. Michels, "Synergies in hemiplegia," Clin. Management, vol. 1, pp. 9-16, 1981.

[21] S. Brunnstrom, Movement Therapy in Hemiplegia. New York: Harper and Row, 1970.

[22] A. R. Fugl-Meyer, L. Jaasco, L. Leyman, S. Olsson, and S. Steglind, "The post-stroke hemiplegic patient," Scand. J. Rehab. Med., vol. 7, pp. 13-31, 1975.

[23] J. P. A. Dewald, P. S. Pope, J. D. Given, T. S. Buchanan, and W. Z. Rymer, "Abnormal muscle coactivation patterns during isometric torque generation at the elbow and shoulder in hemiparetic subjects," Brain, vol. 118 , pp. $495-510,1995$.

[24] J. P. A. Dewald, R. F. Beer, and J. D. Given, "Evidence for task dependent weakness due to abnormal torque synergies in the impaired upper limb of hemiparetic stroke subjects: Preliminary results," in Proc. 18th Annu. Int. Conf. IEEE/EMB Soc., 1996.

[25] D. J. Reinkensmeyer, J. P. A. Dewald, and W. Z. Rymer, "Workspace deficits and abnormal synergies during guided reaching after stroke," Soc. Neurosci. Abstracts, p. 2041, 1996.

[26] D. J. Reinkensmeyer and W. Z. Rymer, "Using a mechanical guide to evaluate and treat workspace deficits after brain injury," in Proc. 19th Annu. IEEE Eng. Med. Biol. Soc. Conf., 1997.

[27] J. Desrosiers, D. Bourbonnais, G. Bravo, P. M. Roy, and M. Guay, "Performance of the 'unaffected' upper extremity of elderly stroke patients," Stroke, vol. 27, pp. 1564-1570, 1996.

[28] R. W. Hamming, Digital Filters. Englewood Cliffs, NJ: Prentice Hall, 1983.

[29] R. M. Murray, Z. Li, and S. S. Sastry, A Mathematical Introduction to Robotic Manipulation. Boca Raton, FL: CRC Press, 1994.

[30] R. Calliet, Shoulder Pain, 3rd ed. Philadelphia, PA: F.A. Davis, 1991

[31] J. H. Carr and R. B. Shepherd, A Motor Relearning Programme for Stroke, 2nd ed. Oxford, U.K.: Butterworth-Heinemann, 1992.

[32] M. L. Aisen, H. I. Krebs, N. Hogan, F. McDowell, and B. Volpe, "The effect of robot-assisted therapy and rehabilitative training on motor recovery following stroke," Arch. Neurol., vol. 54, pp. 443-446, 1997.

[33] H. I. Krebs, N. Hogan, M. L. Aisen, and B. T. Volpe, "Robot-Aided neurorehabilitation," IEEE Trans. Rehab. Eng., vol. 6, pp. 75-87, 1998.

[34] D. J. Reinkensmeyer, J. P. A. Dewald, and W. Z. Rymer, "Robotic devices for physical rehabilitation of stroke patients: Fundamental requirements, target therapeutic techniques, and preliminary designs," Technol. Disability, vol. 5, pp. 205-215, 1996.

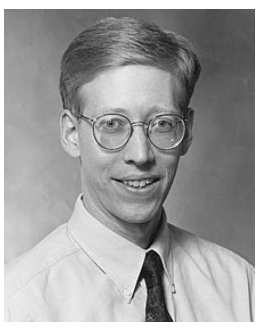

David J. Reinkensmeyer (M'95) received the B.S. degree in electrical engineering from the Massachusetts Institute of Technology, Cambridge, in 1988, and was awarded the Ernst H. Guilleman Prize for best undergraduate thesis in electrical engineering for research in robot learning. He received the M.S. and Ph.D. degrees in electrical engineering from the University of California at Berkeley in 1991 and 1993, respectively, studying neuromuscular control and robotics with support from a National Science Foundation graduate

fellowship.

After lecturing at the University of California at Berkeley in 1994, he received postdoctoral training at the Rehabilitation Institute of Chicago/Northwestern University Medical School, Evanston, IL, as a National Institute for Disability and Rehabilitation Research Training Fellow. In 1996, he received an NIH National Research Service Award from the National Center for Medical Rehabilitation Research to study mechanisms of arm impairment after brain injury. In 1998, he joined the Department of Mechanical and Aerospace Engineering at the University of California, Irvine, as Assistant Professor. His research interests are in medical mechatronics, biomechanics, and neurorehabilitation.

Dr. Reinkensmeyer is a member of the IEEE-EMB Society, the Society for Neuroscience, and RESNA.

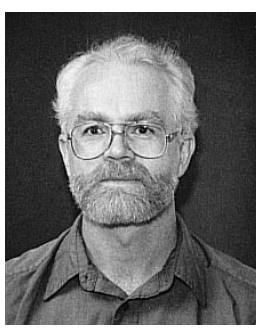

Julius P. A. Dewald received the B.S. and M.S. degrees in physical therapy and rehabilitation medicine from the Free University of Brussels, Belgium, in 1978 and 1980, respectively. After clinical training in neurorehabilitation and appointments as a Clinical Instructor in Europe, he returned to graduate school and received the Ph.D. degree in neurophysiology from Loma Linda University, Loma Linda, CA, in 1992

In 1992, he held a part-time Clinical Assistant Professor position at Loma Linda University School of Allied Health. He finished his postdoctoral training at the Rehabilitation Institute of Chicago, IL, in 1995 and is currently an Assistant Professor in the Department of Physcial Medicine and Rehabilitation and the Programs in Physical Therapy at Northwestern University Medical School, Evanston, IL. He is also Senior Clinical Research Scientist position in the Sensory Motor Performance Laboratory at the Rehabilitation Institute of Chicago. His research interests encompass the study of abnormal motor control following injury to the central nervous system using biomechanics and system identification techniques.

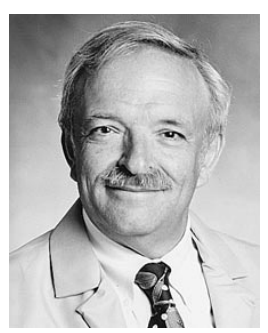

William Zev Rymer (M'92) received the undergraduate and medical training from the University of Melbourne, Australia, graduating with honors in medicine in 1962. After completing his residency in internal medicine, he returned for Doctoral training in the neurosciences and motor control at Monash University, Australia, completing his studies in 1972 .

He received postdoctoral training in the Laboratory of Neural Control in the NIH (1972-1974) and then the Department of Physiology, from the Johns Hopkins University, Baltimore, MD (1974-1976). He is currently the John G. Searle Professor of Rehabilitation Research in the Rehabilitation Institute of Chicago, IL. After two years as an Assistant Professor in Physiology and Neurosurgery at State University of New York at Syracuse, he moved to Northwestern University, Evanston, IL, joining the Department of Physiology in 1978, with joint appointments in biomedical engineering and neurology. In 1989, he assumed the position of Research Director at the Rehabilitation Institute of Chicago accepting an endowed chair. He currently holds faculty appointments in the Departments of Physical Medicine and Rehabilitation, Physiology, and Biomedical Engineering at Northwestern University. $\mathrm{He}$ is also President of the Rehabilitation Institute Research Corporation. His research interests include the neural control and biomechanics of movement in human and animal models, and the disturbances of voluntary movement and their origins in neurologically disabled subjects, particularly those suffering from spinal cord injury and stroke. He currently holds grants from the NIH, NIDRR, the VA, and from several foundations. He has published 102 papers in the fields of biomechanics and motor control.

Dr. Rymer is a member of the ASB, the Society for Neuroscience, the ACRM, the AAP, and EMBS. He has served on the Editorial Board of several journals, including the Journal of Neurophysiology, and has been a member of Musculoskeletal and Orthopedics IRG and the new Geriatrics and Rehabilitation IRG of the NIH. He has served on advisory committees for Paralyzed Veterans of America and for the Canadian Centers of Excellence. He has chaired NIH advisory panels on Rehabilitation Research and has served on Research Committees for the American Congress of Rehabilitation Medicine and for the Association of Academic Physiatrists. 